\title{
Erratum to: Railway restructuring and organizational choice: network quality and welfare impacts
}

\author{
David Besanko1 $^{(D)} \cdot$ Shana $\mathrm{Cui}^{2}$
}

Published online: 3 March 2017

(C) Springer Science+Business Media New York 2017

\section{Erratum to: J Regul Econ (2016) 50:164-206 DOI 10.1007/s11149-016-9309-3}

In our paper (Besanko and Cui 2016), our analysis of the regulator's optimization problem under vertical separation (VS) was incorrect. Our model presumes that the regulator under VS chooses a variable access charge to maximize social welfare. The regulator's problem, stated in Eq. (19) of the paper, imposes the constraint $c \in\left[\eta_{V}, \bar{c}\right]$ on the regulator's choice of the variable access charge $c$. We claimed the constraint was needed to ensure network quality $q_{V}$ was nonnegative and less than its upper bound $\bar{q}$. This was incorrect. This is because we do not need to set $c \geq \eta_{V}$ to ensure that $q_{V} \geq 0$. If the regulator set $c<\eta$, then the network firm would still set $q_{V}=0$. Similarly, if the regulator set $c>\bar{c}$, the network firm would still set quality to $\bar{q}$.

The proper way to state the regulator's problem is without the upper- and lowerbound constraints on $c$. We then can solve this problem by taking into account the properties of the social welfare function $S W(c)$ implied by the piecewise nature of $q_{V}^{*}(c)$ as given in Eq. (14). ${ }^{1}$

\footnotetext{
1 We have redone this analysis, and a detailed explanation of the revised solution is available on request from the authors.
}

The online version of the original article can be found under doi:10.1007/s11149-016-9309-3.

$凶$ David Besanko

d-besanko@kellogg.northwestern.edu

Shana Cui

csnjxl@gmail.com

1 Kellogg School of Management, Northwestern University, Evanston, IL 60208, USA

2 School of Economics and Management, Beijing Jiaotong University, Beijing 100044, China 
The revised analysis yields two insights. First, there is a wider range of parameters where we have a corner solution in which network quality under VS is zero. Second, when network quality is zero under VS, the regulator will set a variable access charge that is less than the marginal cost $\eta_{V}$ of network access. Intuitively, because the regulator is not concerned with inducing the network firm to invest in quality, it uses the variable access charge to counteract completely the downstream market power implied by imperfect oligopoly competition among the transport operators (Laffont and Tirole 2000).

Given these two results, we have redone the propositions in the paper and the computational analysis. With respect to the propositions, (a) Proposition 1 still holds. Moreover, given the revised results, we have an additional condition under which network quality under VS is less than it is under horizontal separation (HS); (b) Proposition 2 requires that we add an additional condition to those stated in the paper. Details are in the Online Errata; (c) Proposition 3 still holds.

In the revised computational analysis, details of which are in the Online Errata we find relative to the published paper (a) the probability of higher quality under VS is smaller; (b) the probability of higher consumer surplus under VS is larger; and (c) the probability of higher social welfare under VS is larger.

Finally, let us summarize how the revised analysis changes our main conclusions, as reported in the introduction of the paper.

- "We find that in general the levels of network quality, consumer surplus, and social welfare arising under VS may be higher or lower than those that arise under HS. Still, there are strong forces at work in our model that tend to make network quality under HS higher than under VS." This insight is still correct.

- "We show that the typically higher quality under HS acts a strong force to push consumer surplus and social welfare under HS to be higher that they would be under VS." This insight is correct but requires more nuance. High quality under HS tends to increase consumer surplus and social welfare under HS, but in our revised analysis, consumer surplus and social welfare under VS can potentially be higher than they are under HS even when VS induces zero network quality. This is because the regulator sets the variable access charge below the marginal cost of network access, inducing first-best prices in the transport market (conditional on $q=0$ ), whereas the transport prices under HS are distorted.

- "... the "best case" for HS from a social welfare perspective occurs when the system can be divided into parallel competing rail systems with just enough geographic differentiation to create moderate, but not cut-throat, competition. Our analysis suggests that under these circumstances there would be a high likelihood that network quality, consumer surplus, and social welfare would all be higher under HS than VS." The advantage that HS enjoyed with respect to consumer surplus for moderate values of the competition intensity parameter $b_{H}$ is less strong than in the published paper. Thus, while moderately positive values of $b_{H}$ may still be the best case for HS from a social welfare and quality perspective, consumer surplus may be lower under HS than under VS under these conditions. The revised analysis illustrates that there is a greater tendency for consumer surplus to be higher under VS than under HS more globally. This is because our orig- 
inal analysis ignored, while our revised analysis incorporates, the flexibility for the regulator to set the variable access charge less than the marginal cost of network access to offset the market power of downstream transport firms. The revised analysis suggests perhaps an even more interesting — and difficult-trade-off for policy designers: HS has a strong tendency for higher network quality, while VS has a moderately strong tendency for lower transport prices and higher consumer surplus.

\section{References}

Besanko, D., \& Cui, S. (2016). Railway restructuring and organizational choice: Network quality and welfare impacts. Journal of Regulatory Economics, 50(2), 164-206.

Laffont, J.-J., \& Tirole, J. (2000). Competition in telecommunications. Cambridge, MA: MIT Press. 